\section{EFECTOS ADVERSOS DE LAS NUEVAS TECNOLOGÍAS Y SU INTERFERENCIA EN EL PROCESO DE APRENDIZAJE}

ADVERSE EFFECTS OF THE NEW

TECHNOLOGIES AND ITS INTERFERENCE IN THE LEARNING PROCESS

David Parra Reyes

davidparrare@gmail.com

\section{RESUMEN}

Este artículo nos permite mostrar en forma descriptiva y analítica, basado en evidencias científicas de carácter social, psicológico y clínico, aquellas repercusiones causadas por el exceso o el abuso de la tecnología digital mediante el uso de celulares o smartphones, tabletas con acceso a la Internet, las cuales traen como consecuencias al niño que lo usa, repercusiones metabólicas como la obesidad, repercusiones funcionales como la fatiga visual, mareos, ansiedad o somnolencia; así como alteraciones psicológicas en las cuales predominan las reacciones de irritabilidad, problemas de conductas (berrinches, agresividad, poca tolerancia a la frustración), insomnio, mayor tendencia a la depresión y hasta suicidios (en la edad adolescente). Todos estos factores a su vez interfieren, en gran magnitud, en su salud integral y en el proceso de aprendizaje escolar.

\section{PALABRAS CLAVE}

Uso de tecnología, smartphones, proceso de aprendizaje, alteraciones funcionales.

\section{ABSTRACT}

This article allows us to show in a descriptive and analytical, based on scientific evidence of a social, psychological and clinical, those impact caused by the excess or abuse of digital technology through the use of cell phones or smartphones, tablets with access to the Internet, the, which bring as consequences to the child who uses it, metabolic repercussions such as obesity, functional repercussions such as visual fatigue, dizziness, anxiety or drowsiness; as well as psychological alterations in which the reactions of irritability predominate, behavior problems (tantrums, aggressiveness, little tolerance for frustration), insomnia, greater tendency to depression and even suicides (in the adolescent age). All these factors in turn interfere, in great magnitude, on their integral health and in the school learning process.

\section{KEYWORDS}

Use of technology, smartphones, learning process, functional alterations.
$\mathrm{E}$ n la actualidad, el uso de las nuevas tecnologías por parte de menores está generando cierto desasosiego social debido a su alta frecuencia.

Las instituciones que vigilan la salud a nivel mundial, dentro de las cuales se encuentra la Organización Mundial de la Salud (OMS), desarrollan e implementan planes y estrategias en toda área de la salud tendientes a la promoción y prevención de las alteraciones propias en salud. Como parte de ello, la Academia Americana de Pediatría y la Sociedad Canadiense de Pediatría realizaron una investigación exhaustiva cuyo resultado señala que los bebés que figuran entre 0 a 2 años no deben tener ninguna exposición a la tecnología, los niños de 3 a 5 años deben tener acceso restringido a una hora al día y los menores de 6 a 18 años deben tener acceso restringido a 2 horas al día (AAP 2001/13, CPS 2010). Estos estudios fueron hechos porque se demostró que existe un desmedido uso de la tecnología tanto en niños como en jóvenes que superan las cantidades mayores a la recomendada ( 4 a 5 veces más), lo que está dando como resultado graves consecuencias y amenazas vitales (Kaiser Foundation, 2010; Active Healthy Kids Canada, 2012).

Mediante esta revisión se quiere generar una reflexión sobre el uso de la tecnología en el desarrollo infantil con respecto al conocimiento de alerta existente en el área investigada y dentro 
del ámbito científico. Por consiguiente, se refiere como conclusión la importancia de prevenir el uso de la tecnología en niños y adolescentes y así protegerlos para evitar daños a su salud y en el ámbito académico.

\section{- Las nuevas tecnologías}

Ante la creciente preocupación sobre el uso de las nuevas tecnologías de los menores, se está haciendo común oír que los menores con frecuencia hacen uso inadecuado, descontrolado e incluso adictivo de las nuevas tecnologías.

Por ello, es primordial comprender que entendemos por nuevas tecnologías. Entendemos como nuevas tecnologías a un grupo amplio y variado de instrumentos, aparatos, equipos, herramientas, canales y soportes dedicados al uso, manejo, presentación, comunicación almacenamiento y recuperación tanto analógica como digital (Villadagos y Labrador, 2009).

Ante el uso de las nuevas tecnologías, se han generado cambios importantes en la vida de las personas y en el funcionamiento de la sociedad en los diferentes ámbitos. Facilitan el acceso de la información en forma inmediata y actualizada y nos permite conocer mejor los entornos próximos, alejados y remotos inaccesibles para la mayoría y, en especial, en el entretenimiento y disfrute del ocio.

El acceso y uso a la tecnología, los dispositivos portátiles (celulares, tablets, juegos electrónicos) han aumentado dramáticamente, especialmente por niños muy jóvenes (Common Sense Media, 2013). Esta dedicación en tiempo y atención ha generado una alerta en los padres y profesores al constatar que muchos menores han disminuido otras actividades consideradas positivas como el leer, el hacer deporte o el salir con amigos.

Desde el año 2005, el Instituto Nacional de Estadística e Informática (INEI) publica el Informe Técnico de Estadísticas de las
Tecnologías de Información y Comunicación en los hogares, el cual está elaborado con las bases de datos trimestrales de la Encuesta Nacional de Hogares - ENAHO. La elaboración de este Informe Técnico obedece a la creciente importancia de las Tecnologías de Información y Comunicación (TIC) en todos los ámbitos de la sociedad.

\section{Cuadro $\mathrm{N}^{\circ} 1$}

Perú: Hogares con acceso a servicios y bienes de Tecnología de Información y Comunicación - TIC Años: 2005 - 2013 (Porcentaje)

\begin{tabular}{|l|c|c|c|c|c|}
\hline Año / Trimestre & $\begin{array}{c}\text { Telefonía } \\
\text { fija }\end{array}$ & $\begin{array}{c}\text { Telefonía } \\
\text { móvil }\end{array}$ & $\begin{array}{c}\text { Televisión } \\
\text { por cable }\end{array}$ & Computadora & Internet \\
\hline \multicolumn{5}{c}{ Indicadores Anuales } \\
2005 & 28,5 & 20,7 & 10,3 & 8,8 & 3,7 \\
2006 & 29,9 & 29,8 & 13,9 & 11,2 & 5,1 \\
2007 & 31,0 & 45,0 & 17,0 & 15,4 & 6,6 \\
2008 & 31,9 & 59,7 & 20,0 & 18,0 & 8,6 \\
2009 & 32,1 & 67,0 & 23,1 & 21,2 & 11,0 \\
2010 & 30,4 & 73,1 & 26,0 & 23,4 & 13,0 \\
2011 & 29,8 & 75,2 & 29,8 & 25,4 & 16,4 \\
2012 & 29,4 & 79,7 & 31,9 & 29,9 & 20,2 \\
2013 P/ & 28,6 & 82,0 & 33,6 & 32,0 & 22,1
\end{tabular}

Fuente: Instituto Nacional de Estadística e Informática - Encuesta Nacional de Hogares.

De cada 100 hogares el $82 \%$, al menos un miembro tiene celular, comparado con el 2005, se incrementó en 61,3 puntos porcentuales. El $28,6 \%$ de los hogares tiene conexión de línea para teléfono fijo. El 33,6\% acceden a televisión por cable. El 32,0\% de los hogares tienen al menos una computadora y el $22,1 \%$ tienen Internet.

Gráfico $\mathrm{N}^{\circ} 1$

Perú: Evolución de los hogares que tienen telefonía móvil y telefonía fija. Años: 2000 - 2013

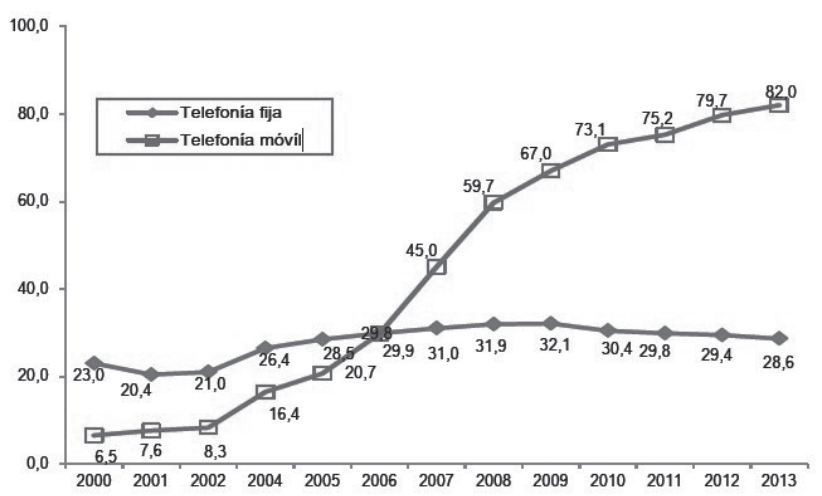

Fuente: Instituto Nacional de Estadística e Informática - Encuesta Nacional de Hogares. 
Cuadro $\mathrm{N}^{\mathrm{o}} 2$

Perú: Población de 6 años y más que hace uso de Internet, según grupos de edad

Años: 2007 - 2013 (Porcentaje del total de población por sexo y grupos de edad)

\begin{tabular}{|c|c|c|c|c|c|c|c|c|c|}
\hline \multirow{2}{*}{ Año / Trimestre } & \multirow{2}{*}{ Total } & \multicolumn{2}{|c|}{ Sexo } & \multicolumn{6}{|c|}{ Grupes de edad } \\
\hline & & Hombre & Mujer & $\begin{array}{c}6 \text { a } 11 \\
\text { años }\end{array}$ & $\begin{array}{c}12 \text { a } 18 \\
\text { años }\end{array}$ & $\begin{array}{c}19 \text { a } 24 \\
\text { años }\end{array}$ & $\begin{array}{c}25 \text { a } 40 \\
\text { años }\end{array}$ & $\begin{array}{c}41 \text { a } 59 \\
\text { años }\end{array}$ & $\begin{array}{l}60 y \\
\text { más }\end{array}$ \\
\hline \multicolumn{10}{|c|}{ Indicadores Anuales } \\
\hline 2007 & 31,1 & 34,9 & 27,2 & 22,6 & 53,2 & 54,6 & 30,5 & 16,0 & 4,0 \\
\hline 2008 & 31,7 & 35,5 & 27,7 & 23,6 & 54,3 & 55,1 & 31,5 & 16,3 & 4,9 \\
\hline 2009 & 33,9 & 37,9 & 29,8 & 26,2 & 56,1 & 57,8 & 34,5 & 18,8 & 5,1 \\
\hline 2010 & 34,8 & 38,9 & 30,5 & 26,4 & 59,0 & 58,7 & 35,2 & 19,3 & 6,0 \\
\hline 2011 & 36,0 & 39,7 & 32,3 & 28,9 & 59,3 & 60,6 & 36,6 & 21,1 & 5,8 \\
\hline 2012 & 38,2 & 41,6 & 34,6 & 29,9 & 60,8 & 64,3 & 39,3 & 24,2 & 7,9 \\
\hline 2013P/ & 39,2 & 42,4 & 36,0 & 31,7 & 61,9 & 66,3 & 41,1 & 24,5 & 8,6 \\
\hline
\end{tabular}

Fuente: Instituto Nacional de Estadística e Informática - Encuesta Nacional de Hogares.

Población adolescente y joven accede más a Internet, $63,5 \%$ y el $65,8 \%$ de la población entre 12 a 18 años y 19 a 24 años de edad, respectivamente, son los mayores usuarios de Internet. Los niños de 6 a 11 años el 32\%. En cambio, la población adulta mayor solo el 8,9\% usa Internet. Cabe destacar, sin embargo, que un $11 \%$ de los menores que reconocen utilizar habitualmente Internet, presenta características como: a) necesidad de conectarse con frecuencia (el 37\% de los encuestados), b) frecuencia elevada de conexión: (el 91\% se conecta más de 10 horas semanales), c) búsqueda de sensaciones, no sólo de información (entre los que necesitan conectarse con frecuencia, el 33\% visita páginas de pornografía, el $40 \%$ páginas de violencia). El $71,8 \%$ de los padres reconocen que en su hogar no hay normas respecto al uso de esta herramienta. El 40,4\% reconoce que, a pesar de que les gustaría saber lo que hacen sus hijos en Internet, les resulta imposible hacerlo.

Gráfico $\mathrm{N}^{\mathrm{o}} 2$

Perú: Población por sexo y grupos de edad, según tipo de actividad que realiza en Internet

Trimestre: Octubre - Noviembre - Diciembre 2013 P/ (\%)

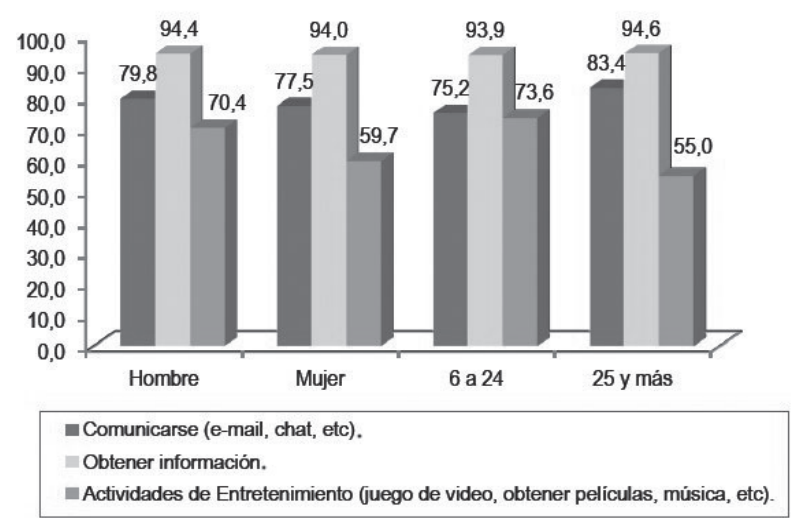

Fuente: Instituto Nacional de Estadística e Informática - Encuesta Nacional de Hogares.
Cuadro $\mathrm{N}^{\mathrm{o}} 3$

Perú: Población que hace uso de Internet, según nivel educativo Trimestre: Octubre - Noviembre - Diciembre 2012 y 2013

(Porcentaje del total de población de 6 y más años de edad de cada nivel educativo)

\begin{tabular}{|l|c|c|c|}
\hline Nivel Educativo & $\begin{array}{c}\text { Oct-Nov- } \\
\text { Dic. 2012 }\end{array}$ & $\begin{array}{c}\text { Oct-Nov- } \\
\text { Dic. 2013P/ }\end{array}$ & $\begin{array}{c}\text { Variación } \\
\text { (puntos } \\
\text { porcentuales) }\end{array}$ \\
\hline Total & $\mathbf{3 9 , 4}$ & $\mathbf{3 9 , 2}$ & $\mathbf{- 0 , 2}$ \\
Primaria 1/ & 16,7 & 16,8 & 0,1 \\
Secundaria & 41,5 & 40,9 & $-0,6$ \\
$\begin{array}{l}\text { Superior no } \\
\text { universitaria }\end{array}$ & 62,4 & 64,9 & 2,5 \\
$\begin{array}{l}\text { Superior } \\
\text { universitaria }\end{array}$ & 85,2 & 85,3 & 0,1 \\
\hline
\end{tabular}

1/ Incluye sin nivel de inicial. P/ Preliminar.

Fuente: Instituto Nacional de Estadística e Informática - Encuesta Nacional de Hogares.

Población con nivel educativo superior accede más a Internet, el $85,3 \%$ de la población con nivel de educación superior universitaria y el $64,9 \%$ con superior no universitaria es usuaria de este servicio; en cambio, solo un $16,8 \%$ de la población con educación primaria o menor nivel accede a este servicio. Debemos tomar en cuenta que los datos cambian rápidamente debido a la implantación progresiva de estos instrumentos.

\section{- Influencia de las nuevas tecnologías en la salud y en el aprendizaje}

\section{Emisión de radiación}

En mayo del 2011, la Organización Mundial de la Salud clasificó los teléfonos celulares (y otros dispositivos inalámbricos) como un riesgo categoría 2B (posible cancerígeno) debido a la emisión de radiación (WHO 2011). James McNamee con Health Canada, en octubre de 2011, emitió un aviso de advertencia diciendo: "Los niños son más sensibles que los adultos a una variedad de agentes -como sus cerebros y sistemas inmunológicos todavía están en desarrollo- entonces usted no puede decir que el riesgo sería igual para un joven adulto cuanto es para un niño" (Globe and Mail de 2011). En diciembre de 2013, el Dr. Anthony Miller, de la Universidad de la Escuela de Salud Pública de Toronto, recomendó que, sobre la 
base de nuevas investigaciones, la exposición a la radiofrecuencia debe ser reclasificada como 2A (probable carcinógeno) y no un $2 \mathrm{~B}$ (posible carcinógeno). La Academia Americana de Pediatría pidió revisar las emisiones de radiación electromagnética de los dispositivos de tecnología citando tres razones en cuanto al impacto sobre los niños (AAP, 2013).

Discutir sobre la relación entre el uso de los celulares y el surgimiento del cáncer cerebral genera una gran polémica. Sin embargo, hay algo en que los científicos concuerdan: los niños son más sensibles a los agentes radiactivos que los adultos; es por ello que investigadores canadienses indican que la radiación de los celulares debería ser considerada como "probablemente cancerígena" para los niños. Independientemente de cuánto los médicos norteamericanos culpan a los celulares y las tabletas por una serie de problemas infantiles, es importante siempre estar atento a los usos de cada aparato. Mantener los electrónicos aliados a la educación de los niños puede ser una salida muy interesante, pero siempre evitando los excesos y la sobreexposición a contenidos agresivos.

\section{$\checkmark \quad$ Uso de pantallas y sus efectos}

El sentido que nos hace recibir la mayoría de la información sobre el entorno que nos rodea es el de la visión. Sin una adecuada salud visual se disminuirá significativamente la capacidad para relacionarse con el entorno, además que una sociedad estará más preparada para afrontar los retos del desarrollo si sus ciudadanos tienen una mejor salud visual.

Laproblemática de las pantallaso video terminales en el ser humano está ligada fundamentalmente con fatiga visual (prurito, lagrimeo, sensación de cansancio, visión borrosa, dolor en el globo ocular), mareos, ansiedad o somnolencia y alteraciones psicológicas (irritabilidad, insomnio, mayor tendencia a depresiones y suicidios).

La utilización de estas tecnologías, como ver televisión, por ejemplo, en especial desde la temprana edad, es un hábito que presenta un alto porcentaje de los niños menores de 4 años. También entre 5 y 9 años y de los niños de 10 años o más cabe acotar que un porcentaje medio de todas estas edades refiere dedicar diariamente y entre semana dos horas o más a esta actividad visual. Respecto al uso de videojuegos, ordenadores o Internet, es un hábito bajo en los menores de 4 años, pasa a un porcentaje medio en la franja de edad de 5 a 9 años y alcanza un pico medio alto en los niños de 10 años o más. Globalmente hay un promedio bajo que refiere dedicar dos horas o más diariamente y entre semana a estas tecnologías. Los porcentajes se elevan durante los fines de semana, sin embargo, un dato mucho más preocupante todavía es que un porcentaje bajo de los niños pasan la tarde solos en casa sin la supervisión de un adulto. Por tal razón y en especial en países desarrollados, han realizado estudios tratando de examinar las consecuencias que su abuso puede tener sobre la salud infantil. En varios estudios han examinado el aumento de los riesgos que contrae la exposición excesiva a los video terminales como son los problemas físicos, el gran impacto negativo a nivel académico y de comportamiento.

\section{- Crecimiento rápido del cerebro y los problemas en su desarrollo}

Los estudios que Christakis realiza en el 2011, indican que entre los 0 y 2 años, el cerebro del niño triplica de tamaño y continúa en estado de rápido desarrollo hasta los 21 años de edad. El desarrollo inicial del cerebro está determinado por estímulos ambientales o en todo caso por la falta de ellos. Se ha demostrado que el desarrollo cerebral con una sobreexposición a las tecnologías (celulares, Internet, iPads, TV) afecta negativamente al funcionamiento y causa déficit de atención, retrasos cognitivos, aprendizaje deficiente, aumento de la impulsividad, disminución de la habilidad de regulación propia de las emociones, por ejemplo: birras (Small, 2008).

Un estudio realizado por Ling-Yi, tomó como muestra un grupo de 75 niños, entre 15 y 35 meses de edad que fueron diariamente expuestos a la 
televisión, un promedio diario de 67,4 minutos de televisión, lo cual según la Academia Americana de Pediatría ya es un tiempo excesivo. Al finalizar el estudio, determinaron que la visualización de la televisión aumentó el riesgo de retraso en el desarrollo cognitivo, lingüístico y motor.

\section{$\checkmark \quad$ Retraso en el desarrollo}

El niño desarrolla habilidades y capacidades mediante el conocimiento de su cuerpo. El uso de la tecnología genera una vida sedentaria lo cual restringe el movimiento, generando un retraso en los menores. Uno de cada tres niños ahora entra en la escuela con retraso de desarrollo, impactando negativamente en la alfabetización y el desempeño académico (Help EDI Maps, 2013). El movimiento aumenta la atención y la capacidad de aprendizaje (Ratey, 2008). Con ello, el uso de tecnología por niños menores de 12 años es perjudicial para el desarrollo del niño y del aprendizaje (Rowan, 2010).

El abuso de la tecnología causa dependencia en los niños; el vicio obstaculiza el desarrollo infantil. La preocupación, por lo tanto, se concentra en los jóvenes y en los niños más vulnerables a este tipo de dependencia, principalmente por el surgimiento cada vez más frecuente de los juegos en línea.

"Cuanto más precozmente ofrezco ese tipo de tecnología a un niño, mayor es el compromiso que va a haber", afirmó, en entrevista al portal Boa Voluntad, el psicólogo Cristiano Nabuco de Abreu, coordinador del Grupo de Dependencias Tecnológicas del Programa Integrado de los Trastornos del Sistema (PRO-AMITI) del Instituto de Psiquiatría del Hospital de las Clínicas de la Facultad de Medicina de la Universidad de São Paulo, pionero en los estudios del tema en Brasil.

A pesar de ser bastante estimulado el uso de la herramienta entre los pequeños, pues auxilian en el desarrollo intelectual, esa utilización tan precoz también trae serios problemas, incluso para el desarrollo neurológico y fisiológico. Varias investigaciones ya mostraron, por ejemplo, que, si se da un texto escrito en papel y el mismo texto en el ordenador, aquellos individuos que leen en la pantalla van a tener un entendimiento perjudicado en casi el 50\% (en comparación a los primeros).

\section{$\checkmark \quad$ Alteraciones visuales}

En la actualidad, investigadores en Corea del Sur realizaron exámenes oftalmológicos en 916 niños de entre 7 y 12 años. De éstas, 60 (6,6\% del total) presentaron características del síndrome pediátrico del ojo seco con base en varias evaluaciones; entre ellas el tiempo de disolución de la lágrima, con la finalidad de probar la estabilidad del canal lacrimal.

En el grupo estudiado, el $97 \%$ de los que presentaron síntomas del síndrome pediátrico del ojo seco informaron que usaron tabletas o smartphones por 3,2 horas al día, en promedio. En contraste, el $55 \%$ de los niños que no presentaron síntomas usaron sus gadgets por sólo 37 minutos al día, además de pasar más tiempo en actividades al aire libre. Otro dato interesante fue que cuando los niños dejaron de usar sus teléfonos durante un mes, los síntomas de ojo seco mejoraron significativamente.

De acuerdo con estudios de la Academia Americana de Oftalmología, cuando miramos a pantallas de smarthphones, tabletas y computadoras por mucho tiempo, por haber menor distancia de visión en pantallas significativamente menores, parpadeamos menos. Por lo tanto, hay un mayor esfuerzo de los ojos y así adquirimos la llamada vista cansada. Esto puede llevar a la evaporación más rápida del canal lacrimal $\mathrm{y}$, consecuentemente, evolucionar hacia el síndrome del ojo seco: cuando la producción de lágrimas es menor de lo que debería y provoca una anomalía en la producción natural de moco y lubricación del órgano de visión. Tal situación, a menudo incómoda, puede afectar negativamente la visión del niño $\mathrm{y}$, consecuentemente, perjudicar su desempeño escolar.

Según el oftalmólogo Luis Eduardo Rebouças de Carvalho, del Consejo Brasileño de Oftalmología, debemos parpadear el mismo número de veces 
que respiramos. Es decir, 16 veces por minuto, en promedio. Quien usa una tableta, por ejemplo, tiene una reducción en el número de parpadeos $\mathrm{y}$, como consecuencia, la cantidad de lágrimas sobre la superficie de la córnea se reduce. Además de la latencia, la visión puede quedar borrosa y parcialmente reducida. "A largo plazo, esto puede provocar cambios en la curvatura de la córnea y hasta cambios en la graduación del ojo", dice.

\section{$\checkmark \quad$ Problemas relacionados al sueño}

El $60 \%$ de los padres no supervisan el uso de tecnología de sus hijos y el $75 \%$ de los niños están autorizados a tener tecnología en sus habitaciones (Kaiser Fundation, 2010). El 75\% de los niños de entre 9 y 10 años son privados de sueño y como consecuencia, sus notas en la escuela son negativamente impactadas (Boston College, 2012).

El uso constante de los aparatos puede generar dependencia en diversos grados. Uno de los mayores problemas relacionados con esto está en el hecho de que muchos niños dejan de dormir para jugar, navegar o conversar en los aparatos. Además de las consecuencias psicológicas causadas por ello, también hay que recordar que la falta de sueño nocturno puede ocasionar problemas en el crecimiento.

La luz emitida por los electrónicos es perjudicial para la calidad del sueño y, consecuentemente, a la salud de un modo general. Para la psicoterapeuta Myriam Durante, el resultado del estudio es preocupante y revela que las noches mal dormidas se están convirtiendo en un problema crónico en Brasil. En el portal Boa Voluntad, ella explicó que "a largo plazo ese joven no va a poder guardar todo lo que procesa durante el día, pues la memoria de larga duración es procesada durante la noche, mientras él duerme. Por eso, él tiene que tener un reposo completo y reparador".

Ella recuerda que dormir mal contribuye, y mucho, a una ganancia acentuada de peso, indisposición para actividades físicas, mal humor, reflejos disminuidos y dolores de cabeza.
"Se quedan sentados todo el tiempo. Ya tenemos casos de trombosis, que sólo aparecía en edad avanzada, ahora surgiendo en adolescentes. Ellos necesitan alimentarse mejor, dormir mejor y hacer más caminatas".

\section{Problemas emocionales}

Las nuevas tecnologías se volvieron una adicción en niños. Estos hábitos tienen efectos positivos como un mejor aprendizaje y desarrollar la creatividad, siempre y cuando su uso sea moderado; pero también tiene efectos negativos.

El excesivo uso de la tecnología es la causa principal de las crecientes tasas de depresión infantil, ansiedad, trastorno de apego, déficit de atención, autismo, trastorno bipolar, psicosis y comportamiento infantil problemático (Bristol, University, 2010; Mentzoni, 2011; Shin, 2011; Liberatore, 2011; Robinson, 2008). Según estudios recientes realizados en Canadá, uno de cada seis niños tiene una enfermedad mental diagnosticada, muchas de las cuales están en uso de medicación psicotrópica peligrosa (Waddell, 2007).

Sin embargo, no es el único país que muestra estos resultados; estudios en diversas partes delmundohan demostrado la relación directa entre la utilización excesiva de tecnología y la existencia de una serie de disturbios emocionales. Entre los más citados por los investigadores están: "Depresión infantil, ansiedad, autismo, trastorno bipolar, psicosis y comportamiento problemático". Es sabido que los niños tienden a repetir comportamientos de los adultos y de todo lo que tomen como referencia, es así que los personajes televisivos o de algún programa por Internet, es también tomado como modelo a seguir. Por lo tanto, la exposición a juegos y películas con violencia excesiva puede causar problemas de agresión también a los niños de hasta 12 años.

El psiquiatra y director del centro de salud mental de Arequipa, Moisés Heresi, José Alvarado Aco, explican que los celulares, tablets, laptops y computadoras atraen por sus colores y brillo. Hay 
niños que pasan más tiempo con estos aparatos que jugando con sus padres.

Alvarado recomienda que menores de 3 a 5 años no pasen más de una hora por día frente a cualquier pantalla, eso incluye la televisión. De lo contrario, hay el riesgo de ludopatía.

El maestro en salud mental, Luciana Nunes, afirma que uno de los puntos más graves del vicio es cuando el uso excesivo de Internet empieza a comprometer las relaciones sociales, afectivas y profesionales. "Hay una falta de interés en actividades fuera de Internet y todo queda un poco aburrido, incluso ir al cine, porque después de todo es más fácil descargar el archivo y verlo solo", destacó en una entrevista con el portal Boa Voluntad.

"La preocupación constante en estar en línea, irritabilidad cuando se le pregunta sobre el tiempo de uso, el uso como medio de huir de problemas emocionales, sociales", son algunos de los síntomas señalados por la psicóloga que retrata la dependencia en Internet. Puede afectar a cualquier persona y a cualquier edad. "El síntoma clásico y lo que llama mucha atención es el compromiso con las actividades de la escuela, pues existe una baja en el desempeño académico, y esos jóvenes prefieren quedarse en las relaciones virtuales que las relaciones presenciales", según la experta, advirtió.

El inicio de esta situación recae en "El síndrome del padre (o madre) del fin de semana". Este fenómeno se define como aquella persona que trabaja de lunes a viernes. El sábado se divierte con sus amigos. Reserva las horas de la mañana y almuerzo del domingo para estar con la familia, señala Alvarado Aco (2014).

La soledad, explica el psiquiatra, es el punto de partida para que los niños y adolescentes se refugien en una pantalla antes que socializar. "Y en el poco tiempo que los padres dedican a sus hijos, se la pasan o comprando más tecnología o revisando el celular. Así toda la familia está abstraída en estos objetos", sostiene.
El Doctor Alvarado alerta que dedicar excesivo tiempo a los juegos de computadora provoca un retraimiento social desde la etapa preescolar. Los niños no juegan con otros menores, tienen problemas en el sueño pues fuerzan su estado de vigilia y se vuelven irritables. Y eso puede devenir en una depresión infantil. Estudios recientes lo han detectado, señala Alvarado. Los menores se vuelven caprichosos, desobedientes e irritables.

También desarrollan ansiedad: presentan pavor nocturno, se comen las uñas, se arrancan cabellos y se los llevan a la boca (tricotilomanía), tartamudez y dejan de controlar sus esfínteres de noche.

Si el pequeño tiene ese padecimiento el tratamiento psicológico no será para él. Son los padres a los que se somete a terapias. "En el 99\% de los casos hemos encontrado que el problema son los padres, no los hijos. Son los padres los que facilitan la tecnología, factores de riesgo para los menores, y son ellos los que dejan de lado a sus hijos", dice tajante el psiquiatra.

Rodolfo Castro, director del Instituto para el Matrimonio y la Familia de la Universidad Católica San Pablo, señala que "Esos aparatos no son los responsables del quiebre de las familias, sino la falta de comunicación de los padres que dedican demasiado tiempo a su trabajo, a sus distracciones y a sus teléfonos".

Aunque en Arequipa no hay un estudio que detalle la situación de la salud mental de la población, la experiencia, dice Alvarado, señala que hace diez años por cada 100 niños se registraban 2 o 3 casos de depresión. Hoy por cada centenar hay entre 15 y 20 pacientes.

\section{$\checkmark \quad$ Agresión}

En el 2007, Anderson indica que el contenido de los medios violentos puede causar agresividad infantil. Los niños están cada vez más expuestos a la creciente incidencia de violencia física y sexual en los medios de hoy. Estados Unidos clasificó la violencia en los medios como un riesgo para la salud pública debido al impacto 
causal sobre la agresión infantil (Huesmann, 2007). La prensa registra aumento del uso de habitaciones de aislamiento con niños que presentan agresividad descontrolada.

\section{$\checkmark \quad$ Obesidad}

Usted ya debe haber escuchado alguna afirmación similar a: "Los niños del siglo XXI forman parte de la primera generación de personas que no van a vivir más que los propios padres". Una de las grandes razones para ello es la obesidad, que puede estar vinculada al uso excesivo de los electrónicos. Se estima que los niños con aparatos en la propia habitación tienen un 30\% más de probabilidad de ser obesos que otros.

El uso de TV y videojuegos está correlacionado con el aumento de la obesidad (Tremblay, 2005). Los niños que poseen dispositivos electrónicos en sus habitaciones tienen un $30 \%$ de aumento en la incidencia de obesidad (Feng, 2011). Uno de cada cuatro canadienses y uno de cada tres niños estadounidenses son obesos (Tremblay, 2011). El 30\% de los niños con obesidad desarrollará diabetes y los individuos obesos tienen un mayor riesgo de accidente cerebrovascular y un ataque al corazón precoz, acortando gravemente la expectativa de vida (Centro para el Control de Enfermedades y Prevención, 2010). En gran parte debido a la obesidad, los niños del siglo XXI pueden ser la primera generación donde muchos no van a vivir más que sus padres (Andrew Prentice, BBC News, 2002).

"Hoy en día, los niños pasan todo el día delante de la televisión o del ordenador y tienen una alimentación con muchas calorías, hay una posibilidad muy grande de que sean obesos", afirmó el endocrinólogo pediátrico Denise Ludovico. Sin contar que esos niños acaban "pareciéndose" a los lechones nada sanos y quedan expuestos a la publicidad de productos alimenticios, induciendo a la ingesta.

Es muy importante que la alimentación sea controlada, pues es en ese momento que el niño necesita recibir vitaminas para fortalecer el organismo, además de ser una fase en la cual los niños crean los hábitos que continúan en gran parte de la vida. Los expertos advierten además que la obesidad es la principal causa de diversas enfermedades en la fase adulta.

Recomendamos que los padres coloquen límites cuando sus hijos asisten y sustituir el tiempo de visualización por oportunidades para la actividad física y educativa.

\section{Demencia digital}

Los psicólogos y los pediatras de los institutos ya mencionados afirman: "Los contenidos multimedia a alta velocidad pueden contribuir a aumentar el déficit de atención". Además, la exposición a esto también causa problemas de concentración y memoria. El motivo para ello sería la reducción de bandas neuronales para la corteza frontal, que ocurre por el mismo motivo recién mencionado (Christakis, 2011). Los niños que no pueden prestar atención no pueden aprender.

\section{Adicciones}

Como los padres se quedan cada vez más atrapados en la tecnología, se están desapegando de sus hijos. En ausencia de apego de los padres, los niños separados pueden conectarse a dispositivos, lo que puede resultar en dependencia (Rowan, 2010). Uno de cada 11 niños con edades entre 8-18 años es adicto a la tecnología (Gentile, 2009).

\section{$\checkmark \quad$ Insostenible}

Las maneras en que los niños son creados y educados con la tecnología ya no son sostenibles (Rowan, 2010). Los niños son nuestro futuro, pero no hay futuro para los niños con sobredosis de tecnología. Cuidar de ello es urgente, necesario $\mathrm{y}$ necesitamos hacer juntos, a fin de reducir el uso de la tecnología por los niños.

A continuación, la Guía de Uso de Tecnología para niños y jóvenes desarrollada por Cris Rowan, terapeuta ocupacional pediátrica y autora del 
Virtual Child (Niño Virtual); Dr. Andrew Doan, neurocientífico y autor de Hooked on Games y el Dr. Hilarie Caixa, Director del reSTART Internet Addiction Recovery Program (Programa de Recuperación de Dependencia de Internet) y autor de Video Games and Your Kids, con la contribución de la Academia Americana de Pediatría y la Sociedad Canadiense de Pediatría, en un esfuerzo por garantizar un futuro sostenible para todos los niños.

\section{$\checkmark \quad$ Guía de uso de la tecnología para niños y jóvenes}

\begin{tabular}{|c|c|c|c|c|c|c|}
\hline Edad & Tiempo & $\begin{array}{c}\text { Tv. no } \\
\text { violento }\end{array}$ & $\begin{array}{c}\text { Dispositivos } \\
\text { de mano }\end{array}$ & $\begin{array}{c}\text { Videojuegos } \\
\text { no violentos }\end{array}$ & $\begin{array}{c}\text { Videojuegos } \\
\text { violentos }\end{array}$ & $\begin{array}{c}\text { violentos en } \\
\text { línea y } \\
\text { pornografía }\end{array}$ \\
\hline $\begin{array}{c}0-2 \\
\text { años }\end{array}$ & Ninguno & Nunca & Nunca & Nunca & Nunca & Nunca \\
\hline $\begin{array}{c}3-5 \\
\text { años }\end{array}$ & 1 hora & $\checkmark$ & Nunca & Nunca & Nunca & Nunca \\
\hline $\begin{array}{c}6-12 \\
\text { años }\end{array}$ & 2 horas & $\checkmark$ & Nunca & Nunca & Nunca & Nunca \\
\hline $\begin{array}{c}13- \\
18\end{array}$ & 2 horas & $\checkmark$ & $\checkmark$ & 30 minutos por día & Nunca \\
años & & & & & \\
\hline
\end{tabular}

\section{CONCLUSIONES}

Una serie de estudios han demostrado que los niños que pasan mucho tiempo frente a las pantallas usando tecnologías basadas en teléfonos celulares tienen mayor riesgo de desarrollar una serie de alteraciones metabólicas, funcionales y psicológicas; las cuales han demostrado que repercuten en el proceso de aprendizaje de aquellos niños que abusan en su uso. Por otro lado, recientes estudios descubrieron que es factor causal del síndrome pediátrico del ojo seco, el cual se caracteriza por la evaporación más rápida del canal lacrimal.

Debido a estas alteraciones y repercusiones frecuentes, a finales del año pasado, la Sociedad Brasileña de Pediatría (SBP) divulgó un manual de orientación para médicos, padres, educadores, niños y adolescentes. El foco es la "Salud de niños y adolescentes en la era digital". El documento inédito en el país fue inspirado en estudios y recomendaciones internacionales y adaptadas a la realidad nacional. Desde entonces, la entidad recomienda que las pantallas estén prohibidas para los bebés de hasta dos años, principalmente en las comidas o antes del sueño. Entre los dos y los cinco años, creen que el límite es que el uso sea de una hora al día. Hasta los seis años, los niños no deben tener contacto con juegos violentos y, hasta los diez años, los niños no deben tener televisión u ordenador en sus propias habitaciones para evitar que sean vulnerables a contenidos inapropiados. 


\section{REFERENCIAS}

Benson V., S., Pirie, K., Schüz, J., Reeves, GK., Beral, V., Green, J. and for the Million Women (2013). Study Collaborators. Mobile phone use and risk of brain neoplasms and other cancers: prospective study. Int J Epidemiol; 42(3), pp. 792-802.

Christakis, D. (2004). Systems Research and Behavioral Science.

Common Sense Media (2013). Cero a ocho: el uso de los medios infantiles en América.

Estadísticas de las Tecnologías de Información y Comunicación en los Hogares. Año: 2007 - 2013 y Trimestre 2011 - 2013. INEI.

Fundación Kaiser (2010). Generación M2: Medios en la vida de 8 a 18 años.

Gentile, R. (2009). Pathological video game use among 8 to 18 year olds.

Grell, K., Frederiksen, K., Schutz, J., Cardis, E., Armstrong, B., Siemiatycki, J. et al. (2016). Intracranial Distribution of Gliomas in Relation to Mobile Phone Exposure: Analyses from the Interphone Study. Am J Epidemiol; 184(11), pp. 818-828.

Help EDI Maps (2013). El Instrumento de Desarrollo Temprano: Informes y Recursos.
Huesmann, L. (2007). The Impact of Electronic Media Violence: Scientific Theory and Research.

Los pros y contra del uso de la tecnología (2016). (Enero 2017). Recuperado de: http://larepublica.pe/sociedad/990364los-pro-y-contra-del-uso-de-1atecnologia-en-los-ninos

Mendieta, Z. (2017). Efectos adversos en el desarrollo visual y cognitivo en niños menores de 3 años relacionados con el tiempo excesivo de uso de pantallas digitales. Tesis de grado. Universidad de la Salle, Facultad de Ciencias de la Salud. Programa de Optometría. Colombia.

Prentice, A. (2002). Obesity's huge challenge for humans. BBC News.

Resultados de la boleta de calificaciones de Active Healthy Kids Canada 2012 sobre la actividad física para niños y jóvenes. Active Healthy Kids Canada 2012.

Rowan, C. (2010). The impact of technology on the developing child.

Tremblay, J. (2011.) Systematic review of sedentary behaviour and health indicators in school-aged children and youth.

Villadangos, S. y Labrador, F. (2009). Anuario de Psicología Clínica y de la Salud, pp. 75-83. 